\title{
Intelligent Fuzzy Enabled Wireless Sensor Network Energy Optimization Scheme using loT
}

\section{RAJU K ( $\nabla$ profgkr@gmail.com )}

EGS Pillay Engineering College https://orcid.org/0000-0001-9434-555X

\section{Lavanya R}

EGS Pillay Engineering College

Kavitha M.G

University College of Engineering Pattukkotai

\section{Radha D}

Arifa Institute of Technology,Esanoor

\section{Research Article}

Keywords: Wireless Sensor Network, WSN, Fuzzy based Neural Data Forwarding Scheme, FNDFS, Internet of Things, loT, Channel Selection, Packet Forwarding

Posted Date: March 16th, 2021

DOI: https://doi.org/10.21203/rs.3.rs-252254/v1

License: (c) (1) This work is licensed under a Creative Commons Attribution 4.0 International License. Read Full License 


\title{
Intelligent Fuzzy enabled Wireless Sensor Network Energy Optimization Scheme using IoT
}

\author{
Raju K ${ }^{1}$, Department of Information Technology E.G.S.Pillay Engineering College,Nagapattinam,India \\ Lavanya $\mathrm{R}^{2}$, Department of Information Technology E.G.S.Pillay Engineering College,Nagapattinam,India \\ Kavitha M.G ${ }^{3}$, Department of Computer Science, University College of Engineering, Pattukkotai, India \\ Radha D ${ }^{4}$ Department of Computer Science, Arifa institute of Technology,Esanoor India
}

\begin{abstract}
In this modern world, energy optimization is the major concern to deal with communication environments especially on Wireless Sensor Networks. Due to the rapid development of Wireless Sensor Network usages, the requirement of energy preservation is also increased in a drastic level. The energy preservation over communication medium is the most important logic to deal with such kind of network based communication model as well as the channel selection and packet forwarding is the most important tasks to take care with such mediums. A Wireless Sensor Networking based channel is considered to be the communication port, in which it takes care of carrying the information and data packets between source and destination entities. The wireless communication channel is containing many important things such as bandwidth, connectivity, connecting wires and so on. The specified things are necessary to carry the data packets between entities, in which the affection on the specified metrics causes the affection on network communications and cause a loss on data packets. The another important constrain is the packet forwarding principles, in which the data packets are moved to the respective selected channel for successful communication as well as the procedure is used to communicate the packets across the region based on the selected channel for communication. These processes are extensively taken care with the Wireless Sensor Network environment management scheme. Several literatures and analysts provide a huge solution to such things, but all are strucked up with certain level of precedence. In this paper, a new methodology is introduced to provide a successful data communication between source and destination based on WSN environment channel selection and packet forwarding principles, the proposed approach is called as Fuzzy based Neural Data Forwarding Scheme (FNDFS). This approach of FNDFS eliminates the unwanted data processing and delays to provide a successful communication between entities using two constraints such as Internet of Things (IoT) and the Fuzzy Logic. The concept of IoT is enabling a internet services to the WSN environment to make the communication between entities without any hurdles as well as the associated functions will be clearly illustrated over the further summaries. These logics are associated together to improve the overall energy efficiency level of the proposed approach in next level. This paper assures the usage of proposed approach FNDFS improves the level of WSN performance based on throughput enhancements, network lifetime improvements, energy efficiency and reduced end-toend delay. These assured things are clearly proved with the graphical results emulated in resulting section of this paper.
\end{abstract}

Index Terms - Wireless Sensor Network, WSN, Fuzzy based Neural Data Forwarding Scheme, FNDFS, Internet of Things, IoT, Channel Selection, Packet Forwarding

\section{INTRODUCTION}

$\mathbf{T}^{\mathrm{n}}$ the communication world, energy efficiency is the critical issue to deal with data transmission between entities. The assistance of Internet of Things (IoT) and the associated developments are rapidly growing in the unexpected level due to its performance and network connectivity support. The association of such IoT with the Wireless Sensor Network medium is so complex in terms of network management as well as the circuit complex level is high. Many past researches works on the analysis of IoT interference with WSN in different variation but all are under certain lacking with energy preservation and time management [1][2]. So, in this paper, the concern is to take care with energy efficiency while communication and the data processing time management in intellectual norms. This paper is intended to design a new framework for Wireless Sensor Network connectivity and energy preservation principles with respect to the adaptation of latest technologies such as Internet of Things. This framework manages the failures and provides a successful communication of data packets between source and destination entities with the association of Internet of Things service enhancements. These features provide an effective data forwarding scheme over WSN communication environment. The association of Internet of Things on WSN environment provides a drastic change in the nature of connectivity as well as the communication level is improved in terms of time and efficiency. The concept of Internet of Things provides range free communication services, so that the network can transmit data 
packets between entities without any range and time considerations [3][7]. The network medium consists of a huge collection of wireless sensor nodes, in which these nodes are configured based on the specific wireless routing protocol definitions such as Adhoc-On-demand Distance-Vector (AODV), Dynamic Source Routing (DSR) and so on. All these protocols are providing a support to nodes to make a successful communication between source node and the destination node in an efficient manner.

However, the range of communication is the more specific constrain in this place, because the transmission efficiency of such communication medium depends on the range and specificity of such channel used for communication. These traditional protocols provide the homogeneous communications in good manner but the range of communication is increased, the level of efficiency of sch protocols is again in a questionnaire level. So, a new communication protocol is required to provide an efficient communication between entities without any range specifications. In this paper, an Internet of Things (IoT) enabled routing protocol is defined, in which it is called intelligent-AODV (iAODV). This proposed protocol changes the parameter metrics in terms of node idleness level reduction, improves the transmission power range (Tx) and the reception power range (Rx). The traditional AODV protocol takes care of associated wireless sensor network communication in an intelligent manner but the complexity level and the processing time is need to be improved in another level of communication such as range free data transmission with selective region perception. For considering such cases the Channel Selection and Packet Forwarding schemes with proposed logics are introduced. The following summary illustrates the channel selection and packet forwarding procedures in clear manner with proper definitions.

\section{A. Channel Selection:}

A hybrid channel selection scheme is applied over the proposed logic of Fuzzy based Neural Data Forwarding Scheme (FNDFS), in which it combines the centralized wireless sensor network architecture as well as the decentralized wireless sensor network scheme. This network architecture based channel selection scheme is based on three distinct metrics such as: bandwidth, data rate and the connectivity. The bandwidth measures are important in the consideration of communication, in which the data packets are communicated between the entities based on the level of available bandwidth in the respective channel. The following equation is considered to estimate the respective bandwidth for communication with respect to the data packets size and distance.

$$
C^{S e l}(x, y) \leftarrow\left(D^{C h a n} \Omega C^{C h a n}\right)+\frac{\operatorname{minCW}}{T P}+\frac{\operatorname{maxCW} \cdot P(x, y)^{2}}{R P}
$$

Where $\mathrm{C}^{\mathrm{Sel}}$ indicates an object for Channel Selection with the ranges of $\mathrm{x}$ and $\mathrm{y}$ region boundaries. $\mathrm{D}^{\mathrm{Chan}}$ and $\mathrm{C}^{\mathrm{Chan}}$ indicates the distributed and centralized channel means, MinCW and $\mathrm{MaxCw}$ indicates the minimum and maximum channel window ranges, $\mathrm{P}$ indicates the probability of collision and TP and RP indicates the transmission and reception probabilities [8]. The following figure, Fig-1 illustrates the channel selection perception in detail with node selection principles based on proposed routing protocol iAODV.

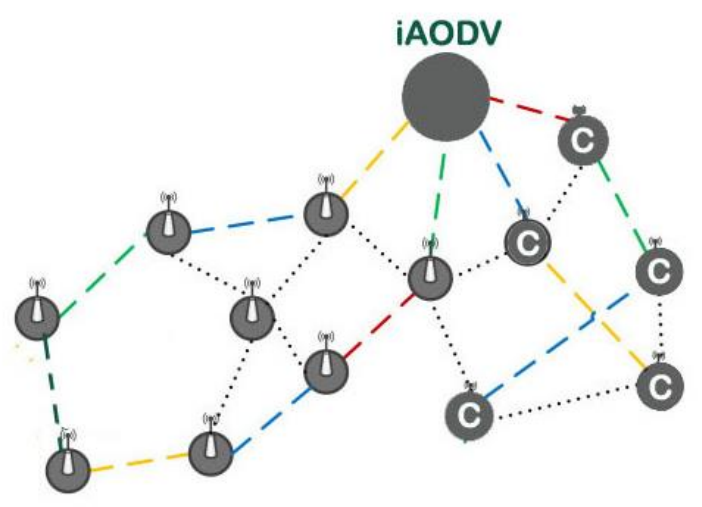

\section{Fig.1 Channel Selection based on iAODV}

The data rate is the concern to mention the speed of data transmission between entities or else it indicates the number of data bits transmitted to the destination per-second. The transmission rate of data is the energy level and the respective speed that a 
specific measure of information is moved throughout a given timeframe and it should realize the exchange rate in case of downloading something on the internet medium or moving information starting with one entity to the other. Initially, transform the data into packets, so that the overall size and transmission force are in either pieces or bytes with a similar prefix (kbps, mbps, gbps or tbps). It can likewise decide the measure of information or the exchange time on the off chance that you know one of the factors in addition to the transmission speed of data packets between entities. The following algorithm, Algorithm-1 is used to indicate the overall flow of channel selection based data rate estimation procedure in detail.

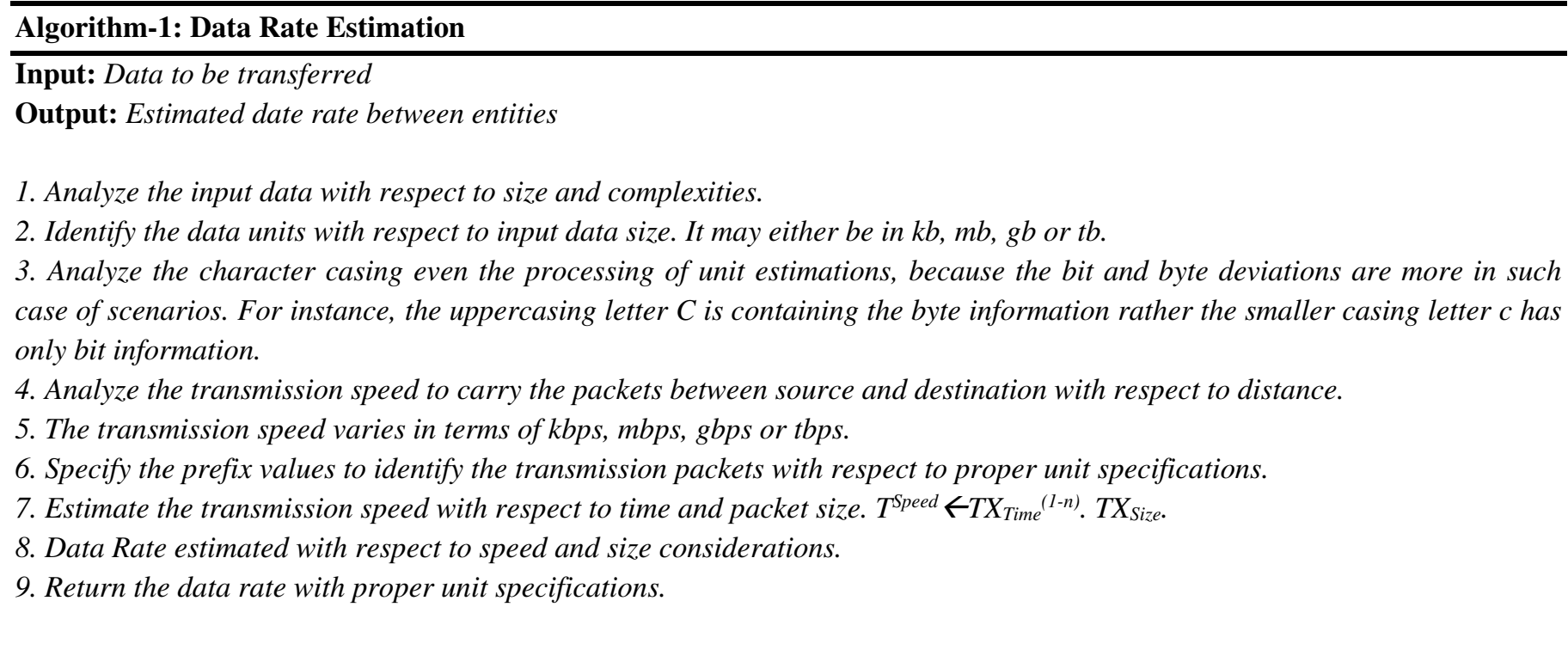

\section{B. Packet Forwarding:}

Packet forwarding is the general term in networking environment, in which it is used to carry the data packets from one end to another end without any data loss assurance. This kind of packet forwarding schemes is categorized into two terms such as centralized packet forwarding and decentralized packet forwarding. In this specification, the centralized packet forwarding features are associated with wireless sensor nodes presented into the centralized routing architecture with range specification and it is also called as localized data packet transmission. In other case, the decentralized approach is quite different with the connected or localized data packet transmission such as it can assist communication between entities without any range specifications. The packet forwarding principles are highly interlinked with the routing terminology, in which the proposed routing protocol called iAODV highly assists the proposed approach of packet forwarding scheme to successfully carry forward the data packets to the respective destinations with respect to the selected data channel. The packet forwarding scheme analyze the routing table and forward the packets to the respective destinations based on the region and distance specified into the routing table. The routes in the wireless sensor network region are considered to be static route, dynamic route and the combined route. The static route is nothing but a default route with common routing protocol specifications, in which it operates while the routing protocols and the associated configurations are defined with static parameters. A dynamic route finding process is quite complex compare to the classical static process, in which the routes are dynamically elected based on the modified routing protocol because the classical and defined routing protocols cannot be customized according to the dynamic principles [9][10]. This paper follows the logic of dynamic routing nature and proposes all logics based on dynamic routing principles. The combined routing strategy is nothing but the combination of both static and dynamic routing principles in fixed range of communications. The following figure, Fig-2 illustrates the proposed approach FNDFS routing protocol iAODV based packet forwarding principles in detail. 


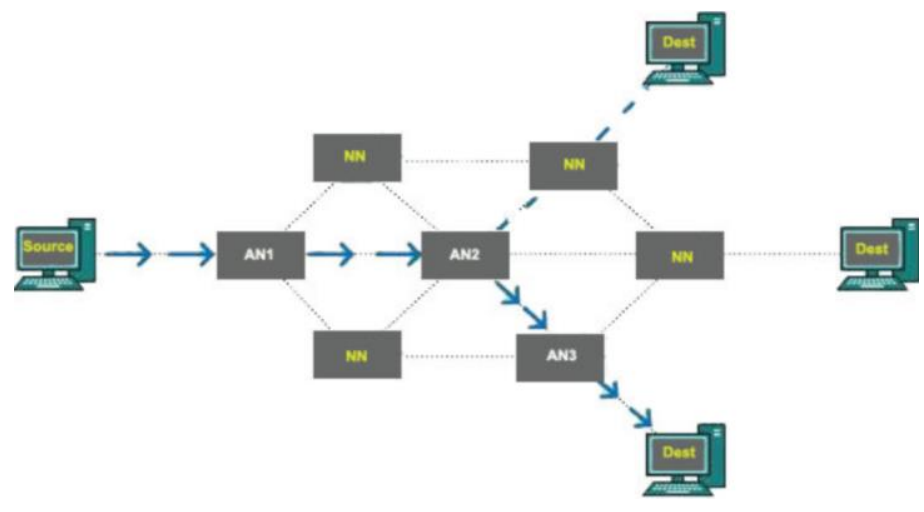

Fig.2 iAODV based Packet Forwarding

In this figure, fig-2 the packet forwarding procedure from source to destination is shown clearly with the indications of 'Dest' indicates the destination node, 'NN' indicates the Neighbor Node and the 'AN' indicates the active neighbor. The following equation demonstrates the principle of packet forwarding with respect to the selected channel, distance between source and destination entities and the routing table metrics.

$$
P(x, y)^{1-n}=\sum_{i=0}^{n-1}\left(\begin{array}{l}
Y \\
X
\end{array}\right) N N^{i} A N^{n-i}
$$

Where the $\mathrm{P}$ indicates the packet forwarding metric with respect to $\mathrm{x}$ and $\mathrm{y}$ range boundaries for all nodes presented into the wireless network region of 1 to $\mathrm{n}$. The following figure, Fig-3 illustrates the proposed approach route selection principles of iAODV, in which the neighbor nodes are electing for forwarding the packets to the destination. However, the active neighbor can only be forward the packets to the next level but the figure portrays the Active Neighbor selection request sending view of the proposed approach.

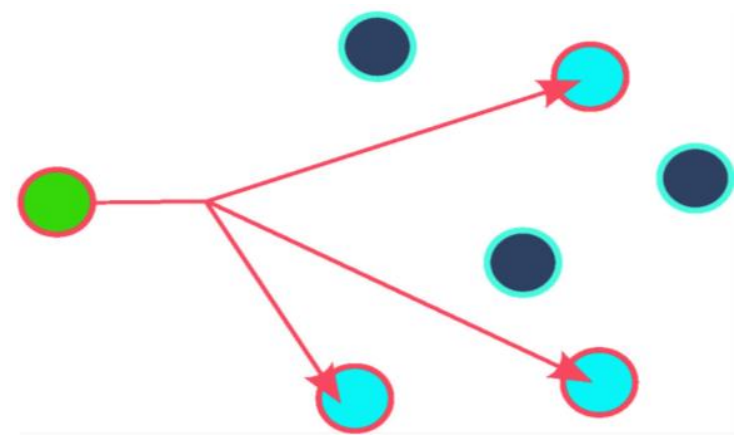

Fig.3 Active Neighbor Selection Request Sending Process

The rest of this paper describe regarding Related Study over section 2, further section of Section 3 illustrates the proposed system methodologies in detail and Section 4 illustrates the Results and Discussion portion of the paper and the final section, Section 5 illustrates the concept of Conclusion and Future Scope of the proposed paper. These all will be explained in detail over the further section summaries.

\section{RELATED STUDY}

In the year of 2020, the authors "VedGautam et al., [4]" proposed a paper related to node localization principles with time synchronization principles based on Small-World Wireless Sensor Network logics. In this paper [4], the authors illustrated such as time synchronized network optimization is the crucial issue in wireless sensor network environment as well as the adaptation of latest technologies such as Internet of Things (IoT0 and the other related technologies creates an issue to deal with WSN platform [4]. This paper [4] concentrates on the network time synchronization problem with respect to network and node 
localization principles. The problems are analyzed over this approach based on the following network metrics such as: network power optimization, bandwidth usages, routing table maintenance and so on. This paper [4] follows multi hop network data transmission model, in which the data from the source entity can able to transfer to the destination via multiple available network hops. The concept of Small-World WSN illustrates the efficiency of node localizations in wireless network environment and the proposed approach of this paper [4] clearly demonstrates the entire view of small-world network schemes in order to limited circumstance of nodes and the associated region formation. The resulting section of this paper [4] estimates the proposed outcome with several classical network formation and localization principles to prove the novelty of the designed approach. This paper [4] proves the proposed approach of Small-World Wireless Sensor Network efficiency in terms of enhanced localization outcome as well as the proposed approach is cross-validated with classical WSN approach to prove the resulting efficiency is better.

In the year of 202, the authors "AbhishekSingh et al., [5]" proposed a paper related to wireless sensor network localization principles with respect to manifold learning associations. In this paper [5], the authors illustrated such as: a new novelty enabled network localization principle is designed to perform an efficient wireless sensor network localization process. This paper [5] adapts manifold learning approach to eliminate or reducing the level of non linear dimensionality as well as the required specification complexity is comparatively high to process the localization process in wireless communication platform. Because of various ecological elements, for example, noisy level and blurring, the inter-node distances determined by the got signal strength pointer are wrong and may not fulfill the localization standard. The wireless sensor nodes are apparently lie on an obscure large dimensionality terms manifold that fulfills the Riemannian-Manifold presumption. Hence, the issue of localization' is changed into the issue of non linear dimensionality decrease and the area of the wireless nodes can be learned through a manifold-learning techniques like 'ISOMAP'. Nonetheless, the directions so acquired should have been changed to get the right areas of the wireless nodes as well as the restricted regions are utilized to examine and adjust the general areas to the supreme regions. The limited portion investigation thinks about every wireless nodes process and the particular boundaries for every sensor nodes dependent on the regions covered by the network. Trial results show that the implemented framework [5] beat past localization' plots and can restrict the wireless sensor nodes with a superior exactness around 31.27 percentages rather than the classical optimization logics.

In the year of 2020, the authors "WahidahMdShah et al., [6]" proposed a paper related to energy efficiency enhancements of the wireless sensor network environment with the association of Internet of Things (IoT) supportivity. In this paper [6], the authors illustrated the details regarding the wireless sensor networks with IoT enhancements. The complexity levels of integrating the wireless sensor network with Internet of Things are huge as well as the sensor nodes are in turn to consume more energy in terms of such associations. This paper provides clustering based energy efficient routing protocol called ImprovedEnergy-Efficient-Clustering-Protocol to enhance the overall wireless sensor network lifetime [6]. The proposed protocol [6] comprises of three different successive parts. Initial, an ideal number of clusters are resolved for the covering adjusted clusters. At that point, the decent static groups are shaped based on enhanced fuzzy $\mathrm{C}$ means logic by consolidating this methodologies with a system to lessen and adjust the energy utilization of the wireless nodes. At last, Cluster-Heads are chosen in ideal areas with turn of the Cluster-Head work among individuals from the cluster dependent on another Cluster-Head determination revolution process by incorporating a back off clock process for Cluster-Head determination and revolution system for ClusterHead turn. Specifically, the proposed convention lessens and balances the energy utilization of nodes by improving the clustering structure, where Improved-Energy-Efficient-Clustering-Protocol is reasonable for networks that require an enhanced lifetime as well as the assessment outcomes demonstrate that the proposed approach [6] performs better than past conventions.

\section{PRoposed System Methodologies}

This paper introduced a new Wireless Sensor Network energy preservation methodology called Fuzzy based Neural Data Forwarding Scheme (FNDFS) in association with the latest communication technology called Internet of Things (IoT). By using this approach of FNDFS, wireless communications between source and destination entities are clear without any failures as well as the energy level preservation is improved in a drastic manner. The proposed approach of FNDFS interfere Fuzzy logic into this system to enhance the resulting accuracy levels by means of utilizing the logic of MinMax procedures. Usually the appliance of fuzzy is to improve the resulting probability as high, as the same manner in this approach of FNDFS utilizes the fuzzy logic to improve the outcome efficiency in terms of energy preservations. The logic of fuzzy implies MinMax procedures to minimize 
the loss ratio and increases the success ratio. The following equation illustrates the logic of fuzzy appliance to attain the maximum probability ratio in outcome.

$$
F z=N_{1-n}+\sum_{i=1}^{n}\left(N t_{(x, y)} \operatorname{Min} \frac{N(1-n)}{D}+\operatorname{Max} \frac{N(1-n)}{D}\right)(3)
$$

Where the Fz represents the fuzzy logic principle enabled variable, $\mathrm{N}$ represents the number of nodes in the wireless network region, Nt represents the Network region range of $\mathrm{x}$ and $\mathrm{y}$ and $\mathrm{D}$ represents the distance between nodes presented into the wireless network environment. The proposed approach of FNDFS introduces a new network routing protocol called intelligentAODV (iAODV), in which it creates a new strategy for communication as well as acquire the neural network principles to process the pathway in an intelligent manner. The appliance of neural schemes generally provides the existing pathway logics based on the traces it collected from the past results. As the Same way in this approach of iAODV accumulates the collected trace values and provides the best pathway to establish the communication between nodes as well as transfer the data packets between source and destination in an efficient manner. The following figure, Fig-4 illustrates the process flow of the proposed approach Fuzzy based Neural Data Forwarding Scheme with respect to iAODV protocol. In this figure, the selection of neighbor nodes are considered under three different scenarios such as energy consumption range, data transfer rate between source and destination entities as well as the interval ratio and the required bandwidth for communication. The neighbor node selection process is handled based on these evaluations and the final selected neighbor node receives the forwarding data to transfer that to the next immediate neighbor. This process continuous until the destination node reached as well as the packets is successfully transmitted to the destination.

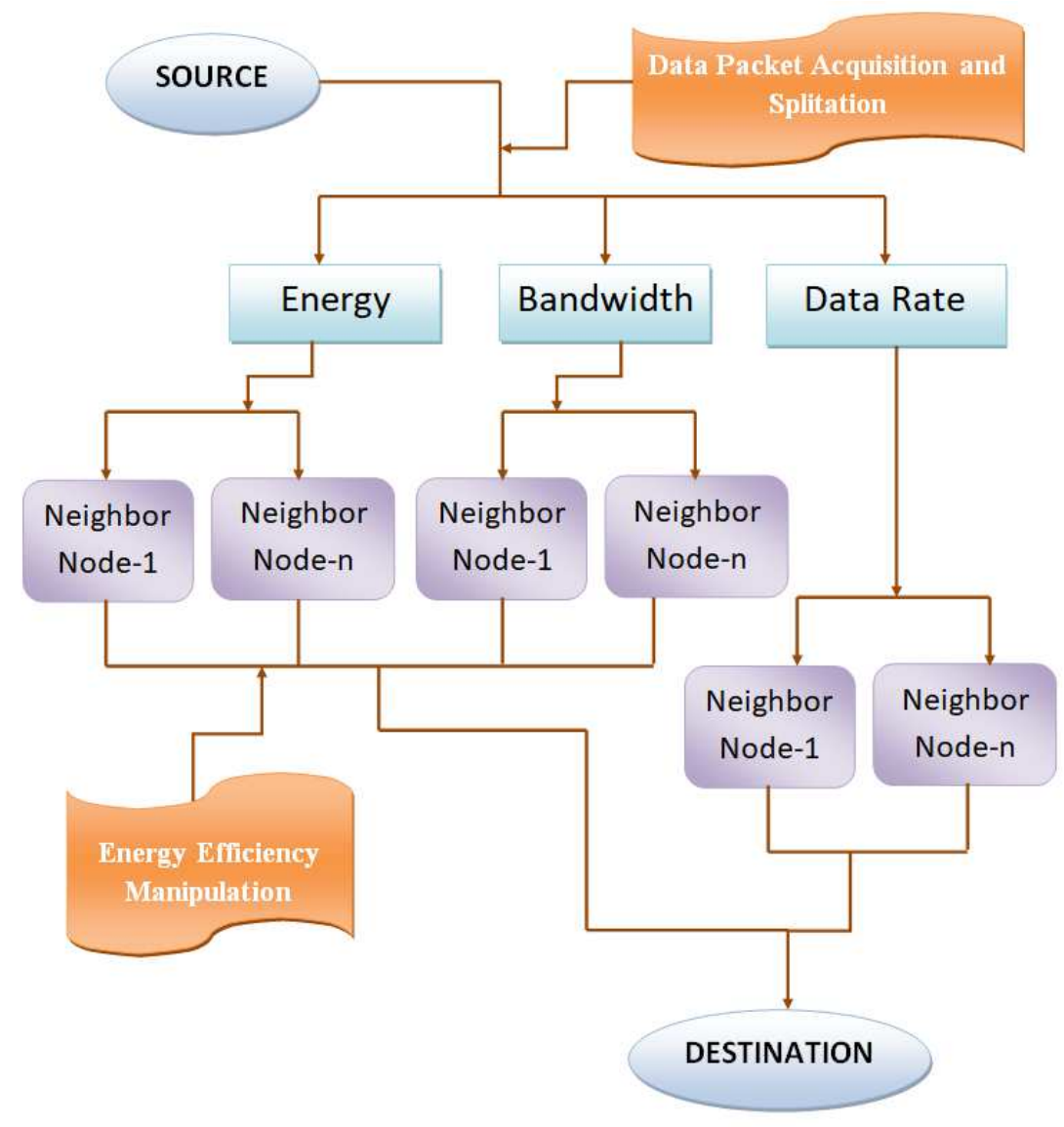

Fig.4 Proposed Approach Process Flow Diagram

The following algorithm, Algorithm-2 illustrates the complete view of the proposed approach of Fuzzy based Neural Data Forwarding Scheme (FNDFS) with respect to the wireless sensor network node configuration in association with intelligentAODV (iAODV) protocol. 


\section{Algorithm-2: Fuzzy based Neural Data Forwarding Scheme}

Input: Data to be transmitted

Output: Successful data transmission improved energy efficiency

1. Node Creation with required input parameters such as packet transmission speed, average interval and so on (ref. Table-1 input parameters).

2. Create an object to establish the wireless environment and adapt the new routing strategy.

3. Define the created protocol On step(2) by means of AdHoc On-demand Distance Vector Routing protocol.

4. Enable the wireless sensor network layer type with respect to Link Layer.

5. Assign the Machine Access Code ratio as 802.11 based IEEE Standard.

6. Create a queue model to dispatch the packets on non-priority manner.

7. Initiate the queue size as customized ranges based on available data to communicate.

8. Two way preoperational antenna is included to provide bi-directional communication abilities.

9. Initiate idle power range of 1.5 milli seconds to improve the energy level.

10. Define the transmission power range ( $T x)$ as $2.5 \mathrm{~J}$ and the reception power range $(R x)$ as 3.5J.

11. Define the sleep ratio of the neighbor node communication model is fixed as $0.000015 \mathrm{~ms}$.

12. Define the initial range energy level of wireless sensor nodes as 200J.

13. Establish the node creation process from 0 to 99 to fix 100 number of nodes into the wireless network environment.

14. Start selecting the source and destination nodes on the created environment.

15. Initiate the communication based on node selections; Fix the neighbor nodes based on energy level occupations in terms of distance and bandwidth efficiency (Ref. Fig-2 and 3).

16. Return the traces to the log file for further references.

\section{RESULTS AND DisCUSSIONS}

In this section, the proposed results are shown with clear specifications as well as the logic of Fuzzy based Neural Data Forwarding Scheme is explained in clear manner with practical outcome scenarios. The proposed appraoch of FNDFS is implemented by using Network Simulator tool, in which the resulting accuracy of the proposed approach as well as the implemented new routing protocol called intelligent-AODV (iAODV) produces the better outcome in resulting units. The following table, Table-1 illustrates the input parameter requirements of the proposed approach with clear range specifications.

Table-1 Input Parameters

\begin{tabular}{|l|l|}
\hline Parameters & Range \\
\hline Number of Nodes & 0 to 99 \\
\hline Node Selection (Source Node) & 1 to 20 \\
\hline Node Selection (Destination Node) & 21 to 99 \\
\hline Packet Size & $\begin{array}{l}1000 \text { to } 2000 \\
\text { kbps }\end{array}$ \\
\hline Individual Node Strength & 100 to $200 \mathrm{~J}$ \\
\hline Transmission Speed & 60 to $65 \mathrm{kbps}$ \\
\hline Time Interval & 0.05 to $0.5 \mathrm{~ms}$. \\
\hline Tx and Rx Power Ranges & $2.5 \mathrm{~J}$ and $3.5 \mathrm{~J}$ \\
\hline
\end{tabular}


The following figure, Fig-5 illustrates the wireless sensor network throughput improvement ratio of the proposed approach FNDFS as well as the throughput enhancements are achieved by means of the following formulation.

$$
T_{h r}(x, y) \leftarrow T_{x}^{P} S+I_{N}{ }^{P}+P_{T} S /\left\{P^{C(1-n)}(i)\right\} \quad(4)
$$

Where the $\mathrm{T}_{\mathrm{hr}}(\mathrm{x}, \mathrm{y})$ indicates the throughput level estimation object, $\mathrm{T}_{\mathrm{x}}{ }_{\mathrm{P}}$ indicates the packet size, $\mathrm{I}_{\mathrm{N}} \mathrm{S}$ indicates the individual node strength, $\mathrm{P}_{\mathrm{T}} \mathrm{S}$ indicates the transmission speed, $\mathrm{i}$ indicates the processing node and the $\mathrm{P}^{\mathrm{C}}$ indicates the power consumption range.

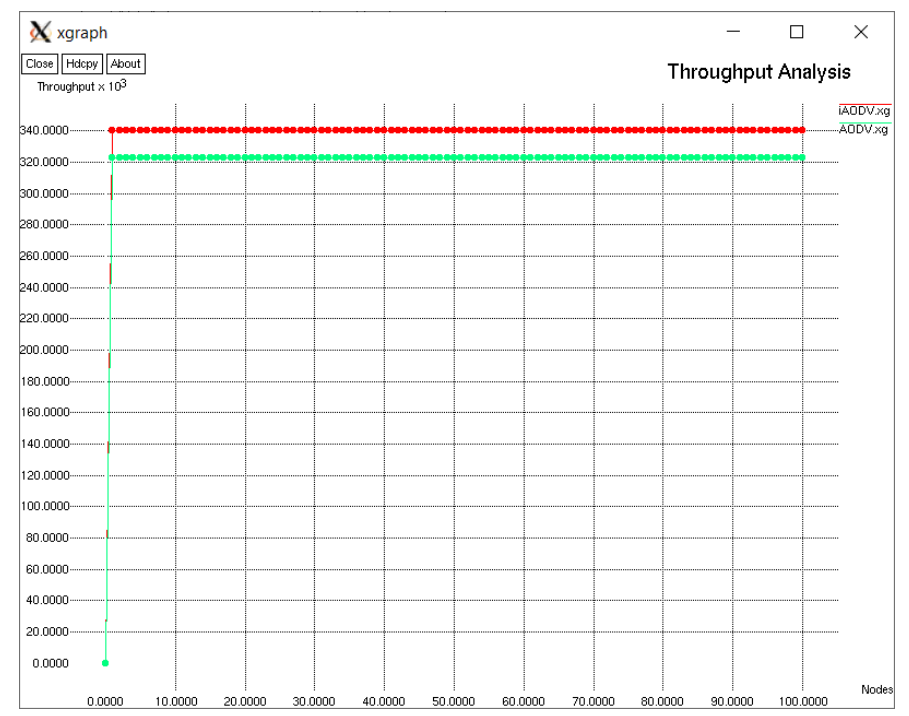

Fig.5 Throughput Analysis

The following figure, Fig-6 illustrates the delay level estimations of the proposed wireless sensor network as well as the delay estimations are achieved by means of the following formulation.

$$
D(x, y) \leftarrow I_{N} S+T_{x}^{P} S /\left(P^{C(1-n)}-T\right)
$$

Where the $\mathrm{D}(\mathrm{x}, \mathrm{y})$ indicates the delay level estimation object, $\mathrm{T}_{\mathrm{x}}{ }_{\mathrm{S}}$ indicates the packet size, $\mathrm{I}_{\mathrm{N}} \mathrm{S}$ indicates the individual node strength, $\mathrm{T}$ indicates the time stamp and the $\mathrm{P}^{\mathrm{C}}$ indicates the power consumption range.

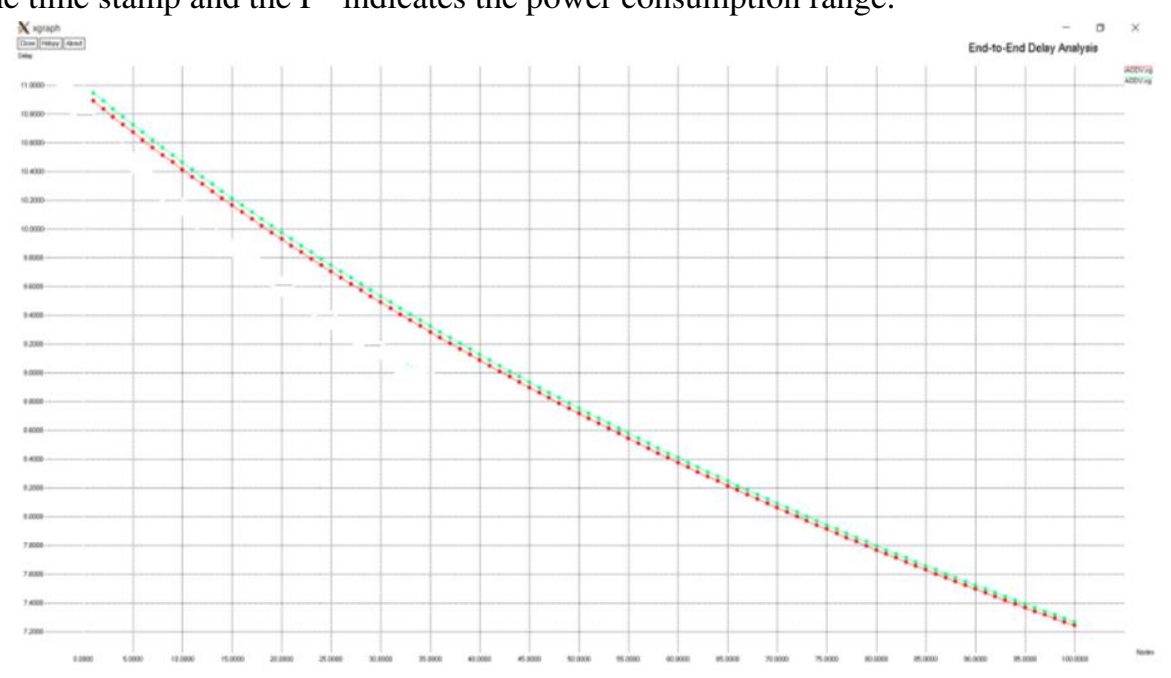

Fig.6 End-to-End Delay Analysis 
The following figure, Fig-7 illustrates the overall lifetime improvements of the proposed wireless sensor network as well as the lifetime enhancements are achieved by means of the following formulation.

$$
L T(x, y) \leftarrow T_{x} P * P^{C(1-n)} * P_{T} S / I_{N} S
$$

Where the $\operatorname{LT}_{\mathrm{r}}(\mathrm{x}, \mathrm{y})$ indicates the overall network lifetime level estimation object, $\mathrm{T}_{\mathrm{x}}{ }^{\mathrm{P}} \mathrm{s}$ indicates the packet size, $\mathrm{I}_{\mathrm{N}}{ }^{\mathrm{S}}$ indicates the individual node strength, $\mathrm{P}_{\mathrm{T}} \mathrm{S}$ indicates the transmission speed and the $\mathrm{P}^{\mathrm{C}}$ indicates the power consumption range.

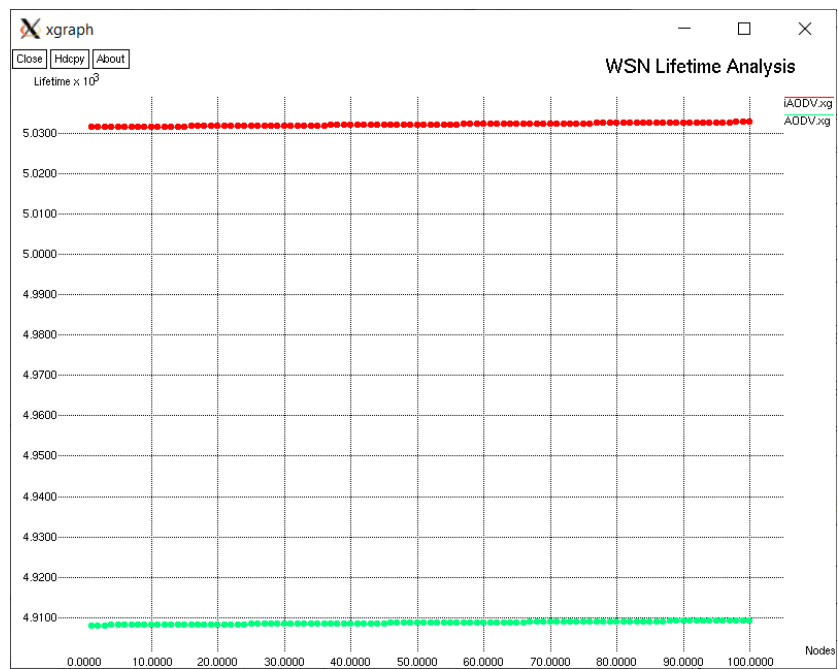

Fig.7 WSN-Lifetime Analysis

The following figure, Fig-8 illustrates the proposed approach energy efficiency level analysis of the wireless sensor network as well as the energy efficiency enhancements are achieved by means of the following formulation.

$$
E_{f f}(x, y) \leftarrow T_{x}^{P} S \sum\left(P^{C(1-n / T)} / P_{T}^{S}\right.
$$

Where the $E_{f f}(x, y)$ indicates the energy efficiency level estimation object, $T_{x}{ }^{P}$ indicates the packet size, $I_{N} S$ indicates the individual node strength, $\mathrm{P}_{\mathrm{T}} \mathrm{S}$ indicates the transmission speed and the $\mathrm{P}^{\mathrm{C}}$ indicates the power consumption range.

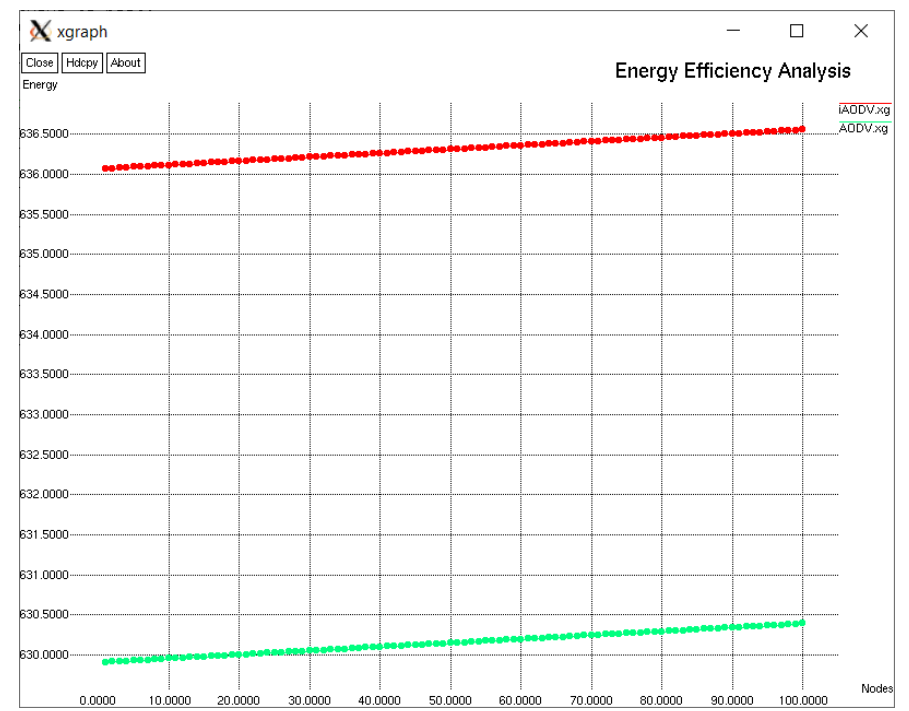

Fig.8 Energy Efficiency Analysis 


\section{CONCLUSION AND FUtURE SCOPE}

In this paper, a novel fuzzy based neural architecture is introduced with the help of classical AODV protocol alterations, in which the proposed approach is named as Fuzzy based Neural Data Forwarding Scheme (FNDFS) and the enhanced AODV protocol is noted as intelligent-AODV (iAODV). These associations produce the best result in outcome as well as the throughput efficiency of the proposed approach is high compare to the classical AODV approach, in which the resulting section figure, Fig-5 illustrated that in a clear manner with proper formulations. The proposed approach improves the energy efficiency level by means of metric formulations defined in figure, Fig- 8 on resulting unit. This paper stands on the commitment and completes the assured efficiency levels in both delay reduction as well as the lifetime improvements by means of the proposed iAODV routing protocol strategy. The resulting section figures, Fig-6 and Fig-7 illustrated that in a clear manner with graphical proof with proper formulations. For all the proposed approach of Fuzzy based Neural Data Forwarding Scheme achieves high accuracy rates on data communication between entities and the resulting section proves the mentioned things in a proper way.

In future the work can further be extended by means of IoT based deep learning metrics to improve the time efficiency as well as the logic of deep learning procedures concentrates on node failures in an intelligent way by means of analyzing the trained dataset model. In which the dataset mentioned is the past tracing records generated from the system logs, by cross-verifying those values and provide the exact predictions of node failures and take appropriate remedies without any hurdles.

\section{Declarations}

Author contributions

All authors contributed to the study conception and design. Material preparation, data collection and analysis were performed by Raju K, Lavanya R, Kavitha M.G and Radha D. The first draft of the manuscript was written by Raju K and all other authors read and approved the final manuscript.

Funding

The authors did not receive support from any organization for the submitted work.

Conflicts of interest/Competing interests

The authors have no conflicts of interest to declare that are relevant to the content of this article.

Availability of data and material

The authors confirm that the data supporting the findings of this study are available within the article.

Code availability (software application or custom code)

The authors confirm that the algorithm and code supporting the findings of this study are available within the article.

\section{REFERENCES}

[1] Hongbo Zhu and Minzhou Luo, "Hybrid Robust Sequential Fusion Estimation for WSN-Assisted Moving-Target Localization With Sensor-Node-Position Uncertainty", IEEE Transactions on Instrumentation and Measurement, 2020.

[2] L.-L. Hung, F.-Y. Leu, K.-L. Tsai and C.-Y. Ko, "Energy-Efficient Cooperative Routing Scheme for Heterogeneous Wireless Sensor Networks", IEEE Access, 2020.

[3] J. Uthayakumar, Mohamed Elhoseny and K. Shankar, "Highly Reliable and Low-Complexity Image Compression Scheme Using Neighborhood Correlation Sequence Algorithm in WSN", IEEE Transactions on Reliability, 2020.

[4] Om Jee Pandey, Ved Gautam, Saket Jha, Mahendra K. Shukla and Rajesh M. Hegde, "Time Synchronized Node Localization Using Optimal H-Node Allocation in a Small World WSN",IEEE Communications Letters, 2020.

[5] Adarsh Prasad Behera, Abhishek Singh, Shekhar Verma and Manish Kumar, "Manifold Learning With Localized Procrustes Analysis Based WSN Localization", IEEE Sensors Letters, 2020. 
[6] Ali Abdul-Hussian Hassan, Wahidah Md Shah, et al., "An Improved Energy-Efficient Clustering Protocol to Prolong the Lifetime of the WSN-Based IoT", IEEE Access, 2020.

[7] Jiahong Chen, Teng Li, Jing Wang and Clarence W. de Silva, "WSN Sampling Optimization for Signal Reconstruction Using Spatiotemporal Autoencoder", IEEE Sensors Journal, 2020.

[8] Sheng-Kai Yang, Ya-Ming Shiue, Zhi-Yuan Su, I-Hsien Liu and Chuan-Gang Liu, "An Authentication Information Exchange Scheme in WSN for IoT Applications", IEEE Access, 2020.

[9] Kawsar Ali and Daniel J. Rogers, "An Orientation-Independent Multi-Input Energy Harvesting Wireless Sensor Node", IEEE Transactions on Industrial Electronics, 2020.

[10] Yu Han, Gang Li, Rui Xu, Jian Su, Jian Li and Guangjun Wen, "Clustering the Wireless Sensor Networks: A MetaHeuristic Approach", IEEE Access, 2020. 
Figures

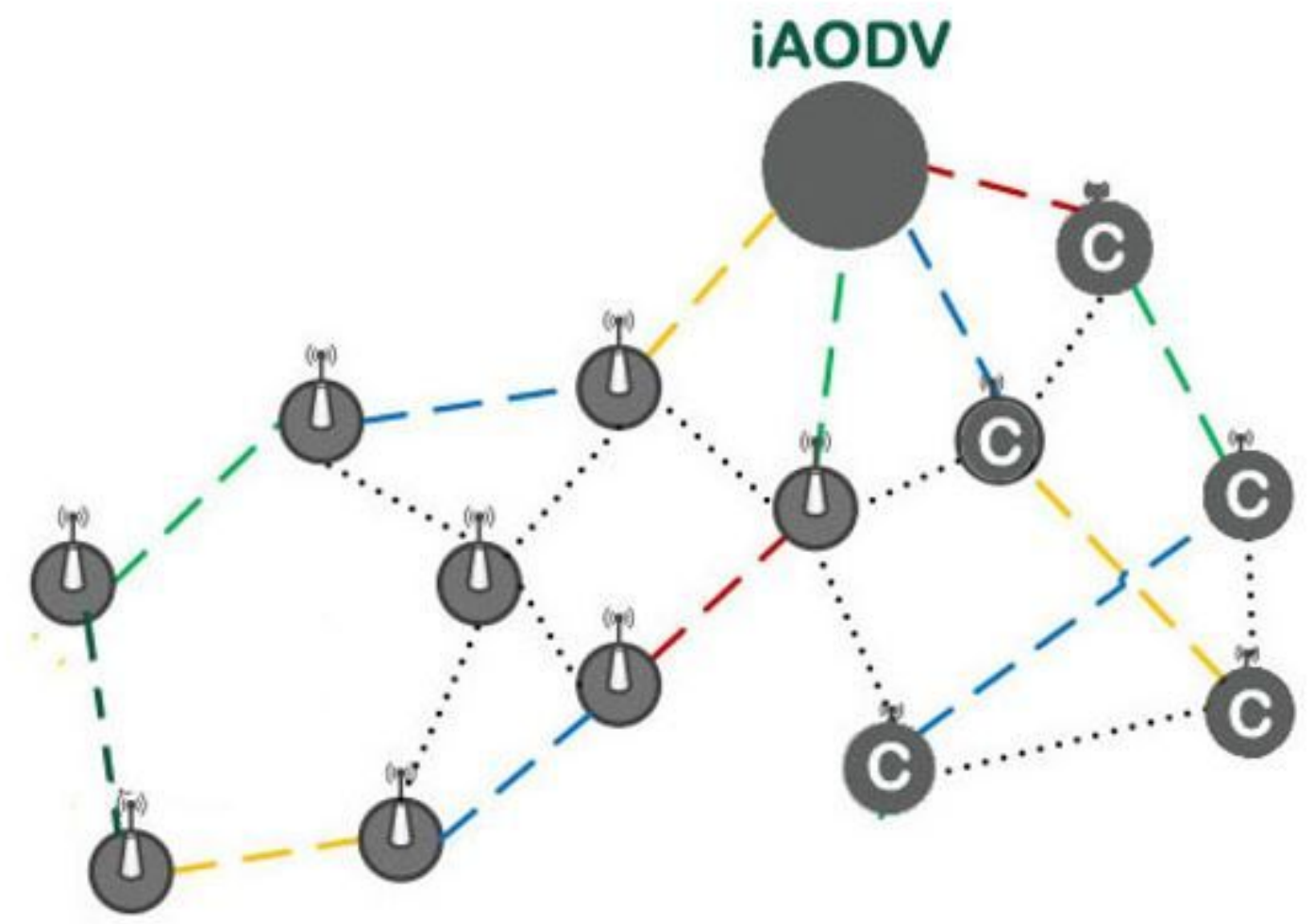

Figure 1

Channel Selection based on iAODV 


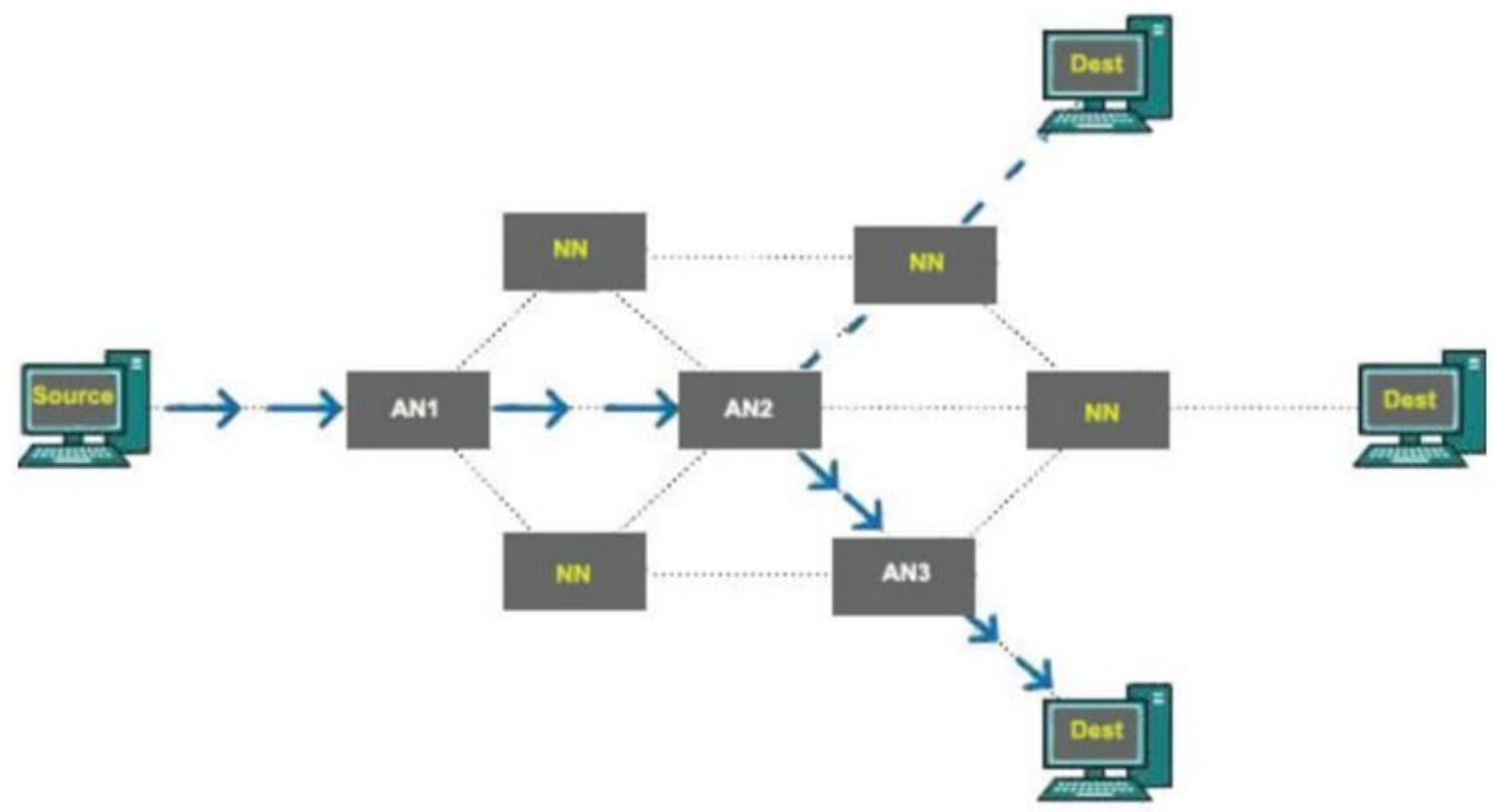

Figure 2

iAODV based Packet Forwarding

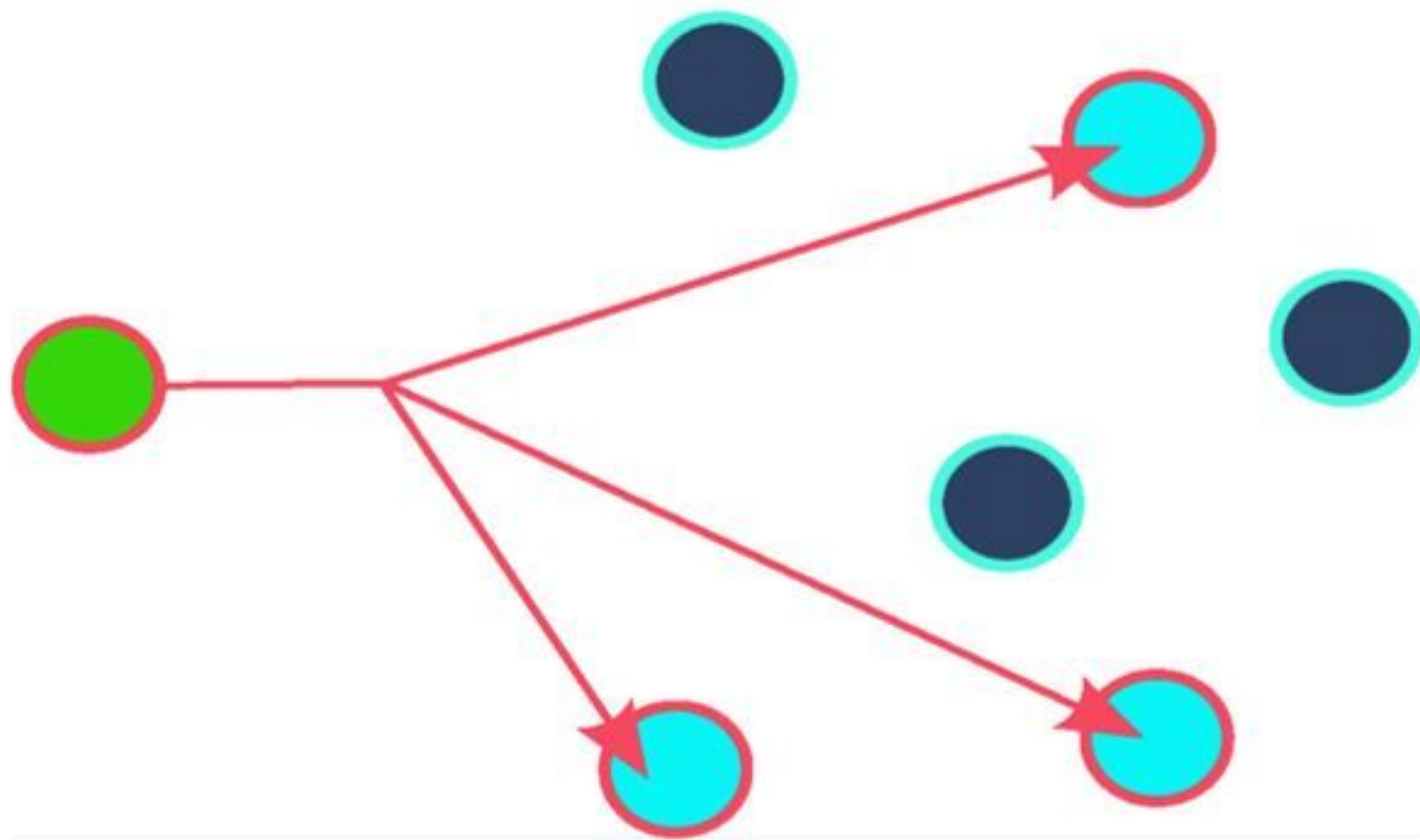

Figure 3 
Active Neighbor Selection Request Sending Process

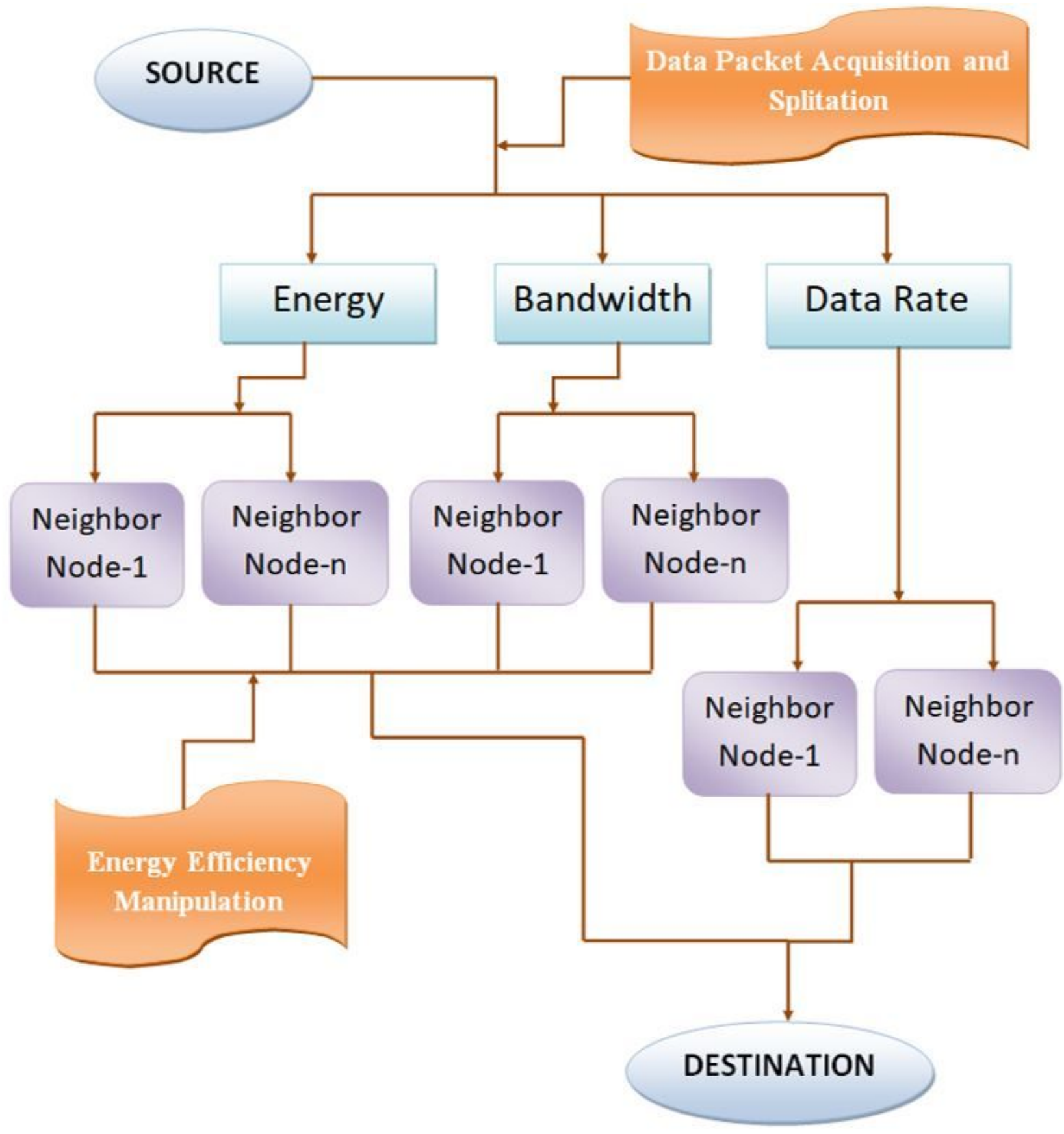

Figure 4

Proposed Approach Process Flow Diagram 


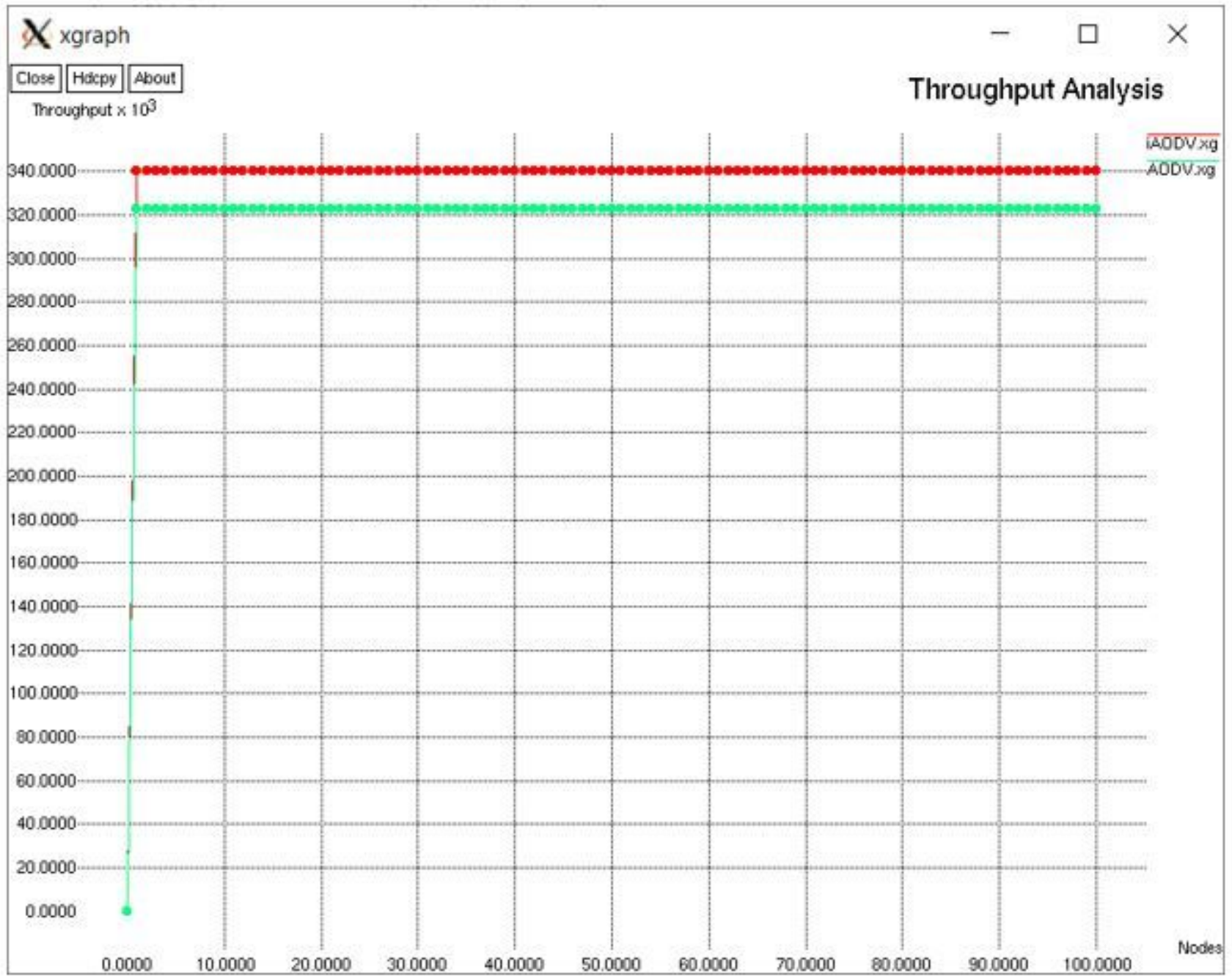

Figure 5

Throughput Analysis 


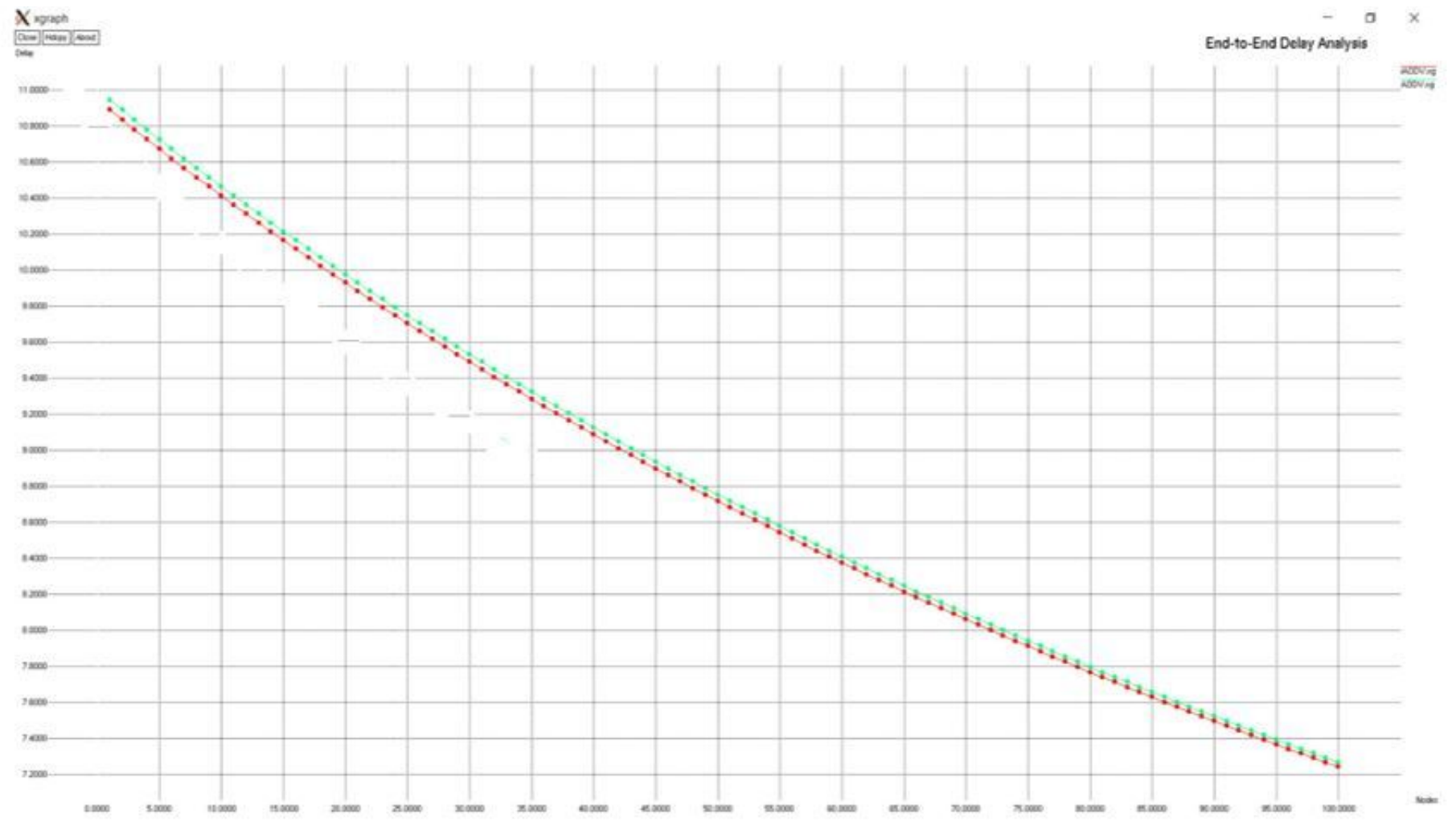

Figure 6

End-to-End Delay Analysis 


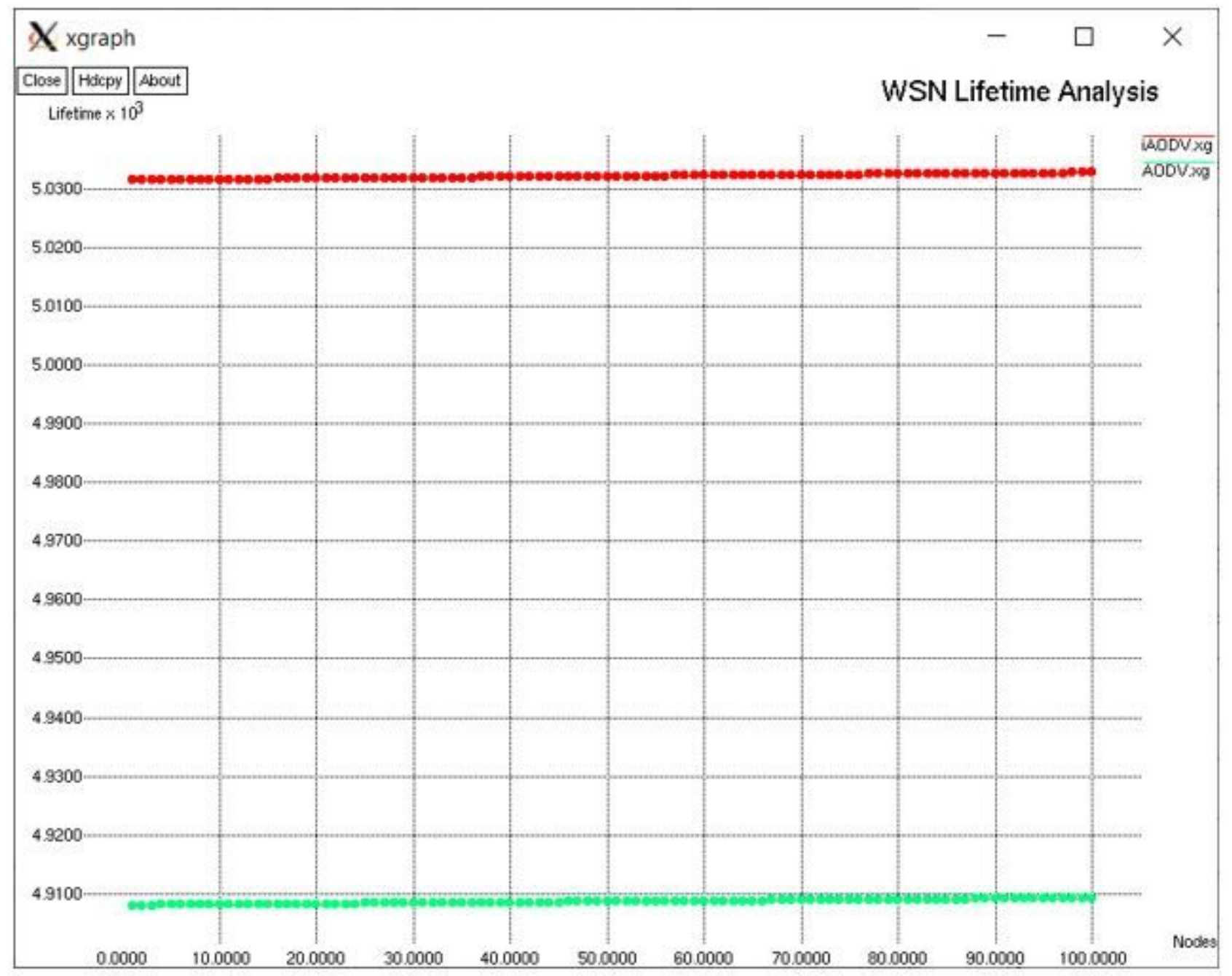

Figure 7

WSN-Lifetime Analysis 


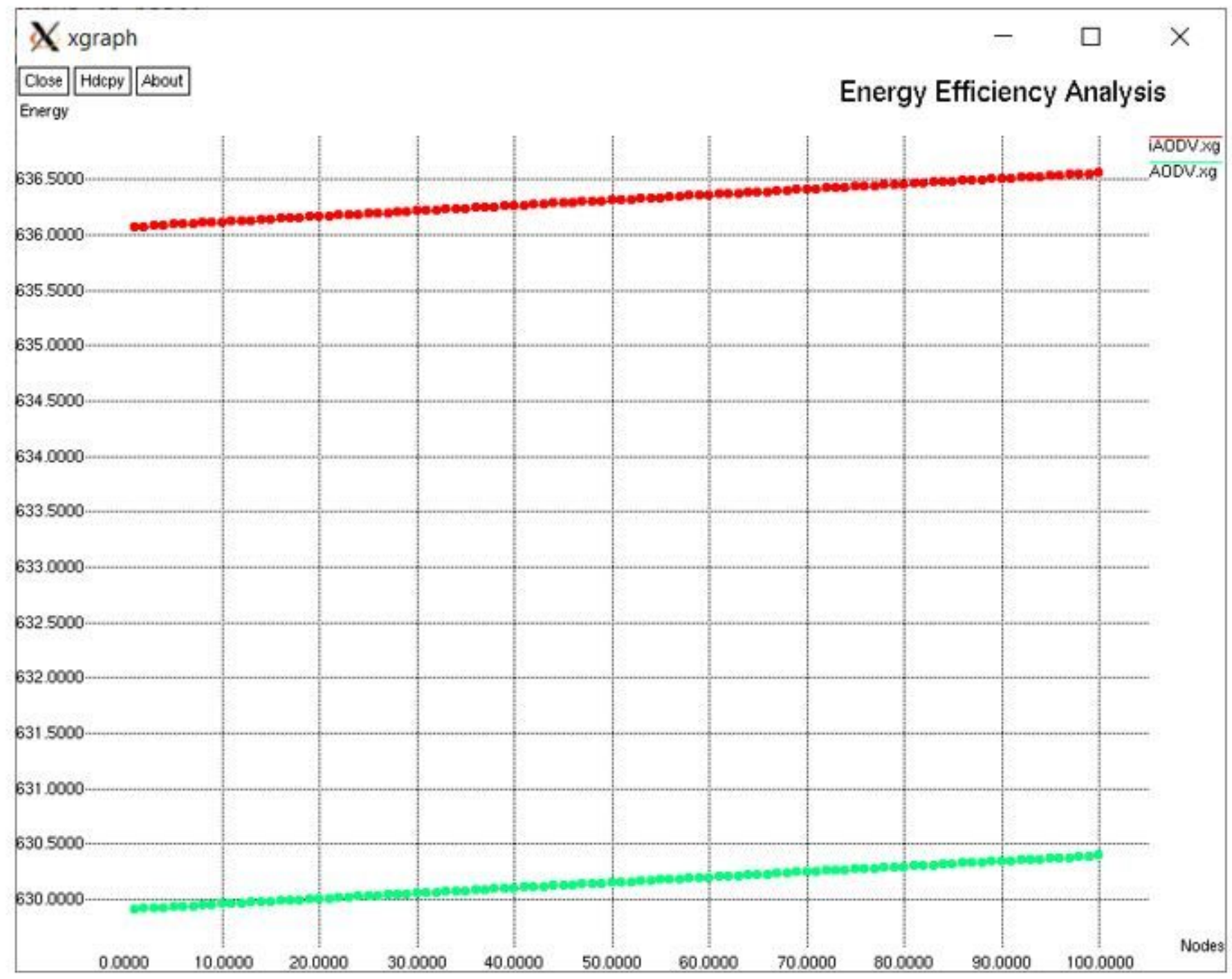

Figure 8

Energy Efficiency Analysis 